\title{
Insegurança alimentar grave municipal no Brasil em 2013
}

\author{
Severe food insecurity in Brazilian Municipalities, 2013
}

Muriel Bauermann Gubert ${ }^{1}$

Rafael Perez-Escamilla ${ }^{2}$

${ }^{1}$ Universidade de Brasília. Campus Darcy Ribeiro. 70910-900 Brasília DF Brasil.murielgubert@gmail. com

${ }^{2}$ Yale School of Public

Health. New Haven

Connecticut EUA.
Abstract The scope of this article was to estimate the prevalence of severe food insecurity (SFI) in Brazilian municipalities in 2013. A logistic regression model was used to predict SFI. To construct the model, the 2013 National Household Sample Survey (PNAD) was used. SFI was measured using the Brazilian Food Insecurity Scale (EBIA). The final model was applied to the 2010 Census, predicting the municipal prevalence. The highest prevalence values were concentrated in the North and Northeast of Brazil, which also showed the highest municipality prevalence disparities. The highest municipal prevalence value of SFI was in the state of Maranhão and the lowest in Rio Grande do Sul. Maranhão was also the State with the largest intrastate disparities in the prevalence of SFI. Spatial analysis showed a higher prevalence of SFI in the North and Northeast regions. Acre had $100 \%$ of its municipalities presenting a very high prevalence of SFI. By contrast in the State of São Paulo, 59.1\% of the municipalities have a low prevalence of SFI. The prevalence of municipal SFI was higher in Brazil's North and Northeast and there were major disparities in intraregional and intrastate distribution. These prevalence values could assist in improving the targeting of policies to combat food insecurity in Brazil.

Key words Food and nutritional insecurity, Hunger, Cities, Prevalence
Resumo O objetivo deste artigo é estimar as prevalências de insegurança alimentar grave (IAG) para municípios brasileiros, em 2013. Construido modelo de regressão logística preditor de IAG, utilizando a Pesquisa Nacional por Amostras de Domicílios (PNAD) 2013. A IAG foi aferida pela Escala Brasileira de Insegurança Alimentar (EBIA). O modelo foi aplicado ao Censo de 2010, sendo preditas as prevalências municipais. As maiores prevalências estão concentradas na Região Norte e Nordeste, que apresentaram também as maiores discrepâncias municipais. A maior prevalência municipal de IAG foi no Maranhão e a menor no Rio Grande do Sul. O Maranhão foi também o Estado com maior discrepância intraestadual na prevalência de IAG. Na análise espacial verificouse que as maiores prevalências de IAG estavam concentradas na Região Norte e Nordeste e se reduzia à medida que desloca-se para o Sul do país. No Acre, $100 \%$ dos municípios apresentaram prevalência muito alta de IAG. Em São Paulo, 59,1\% dos municípios tiveram prevalências baixas de IAG. As prevalências de IAG municipais foram mais elevadas nas Regiões Norte e Nordeste que apresentaram grande discrepância de distribuição intrarregional e intraestadual. Tais prevalências podem auxiliar o processo de melhoria e focalização das políticas de combate à fome no Brasil.

Palavras-chave Segurança Alimentar e Nutricional, Fome, Cidades, Prevalência 


\section{Introdução}

Em 2000, o Brasil foi signatário dos Objetivos de Desenvolvimento do Milênio. O primeiro objetivo previa a redução pela metade da fome e pobreza extrema em 2015, em relação aos níveis de 1990. O Brasil reduziu a extrema pobreza de 25,5\% em 1990 para 3,5\%, em 2012, superando a meta estabelecida ${ }^{1}$. Em 2016, os ODM foram substituídos pelos Objetivos de Desenvolvimento Sustentável que prevê, no Objetivo 2 (Erradicação da Fome), até 2030 acabar com a fome e garantir o acesso de todas as pessoas a alimentos seguros, nutritivos e suficientes durante todo o ano ${ }^{2}$.

A fome no Brasil tem se reduzido nos últimos $\operatorname{anos}^{3}$. A redução da Insegurança alimentar (IA) está associada a melhorias nas condições socioeconômicas da população, bem como à expansão de programas de transferência de renda ${ }^{4}$. Entretanto, ainda assim, 22,6\% da população experimenta algum tipo de IA, caracterizada pela restrição quali-quantitativa da dieta e, em casos mais severos, pela presença de fome ${ }^{3}$.

Considerando-se a insegurança alimentar grave (IAG) como proxy de fome $\mathrm{e}^{5}$ observa-se que, em 2004, 6,9\% da população estava nessa condição, sendo que, em 2013, esse percentual reduziu-se para 3,2\% $\%^{3}$. Entretanto, a distribuição da IAG no território brasileiro não é uniforme. Enquanto na Região Nordeste a prevalência de IAG é de $6,7 \%$, na Região Sul é de apenas 1,9\%. No Estado do Acre ela é de $11,2 \%$, enquanto que no Distrito Federal é de apenas 1,5\%. A desigualdade na distribuição da IAG no Brasil acompanha as tendências de iniquidades sociais ${ }^{3}$.

Essa distribuição heterogênea da IAG entre as regiões, Unidades da Federação (UF) e situação urbana/rural também pode ser evidenciada em nível municipal, menor nível de desagregação política brasileira. Em 2004, Gubert et al. ${ }^{6}$ verificaram que essa diferença municipal variou de $1,0 \%$ a $31,1 \%$ no Brasil. Vianna e Segall-Correa ${ }^{7}$, estudando municípios da Paraíba, também observaram essa mesma heterogeneidade. Portanto, ao analisar-se a situação brasileira, é imprescindível considerar-se uma distribuição díspar da IAG.

Infelizmente, dados municipais não estão disponíveis no Brasil a partir de pesquisas nacionais. A Pesquisa Nacional por Amostra de Domicílios (PNAD), que normalmente agrega o módulo de aferição de segurança alimentar pela Escala Brasileira de Insegurança Alimentar (EBIA), só permite desagregação até UF(3). Dados municipais de IA em municípios brasileiros são oriundos de poucas pesquisas pontuais ${ }^{8-11}$ muitas vezes com recortes populacionais que não permitem a visualização plena da situação do município ${ }^{12-14}$.

Em 2010, Gubert et al. ${ }^{15}$ publicaram metodologia que permite a estimativa de IAG em municípios brasileiros a partir de dados de duas bases nacionais, a PNAD e o Censo Demográfico. Na ocasião, foram geradas estimativas municipais para 5507 municípios brasileiros no ano de 2004 .

Conhecer as prevalências municipais de IAG é importante, uma vez que o município é a unidade executora das politicas publicas vigentes no país. Conhecer a realidade municipal permite o melhor planejamento, implantação, fortalecimento e focalização das politicas publicas de combate à fome no Brasil ${ }^{16,17}$. Esse trabalho tem como objetivo estimar as prevalências municipais de IAG para os 5565 municípios brasileiros no ano de 2013, e analisar a heterogeneidade de distribuição da IAG no país.

\section{Métodos}

Para este estudo utilizou-se dados da PNAD 2013 e o Censo Demográfico Brasileiro, realizado em 2010 (Censo 2010) ${ }^{3,18}$. A PNAD 2013 é a mais recente pesquisa com representatividade nacional com dados disponíveis sobre IA. O Censo 2010 é o mais recente recenseamento da população brasileira. Ambas as bases de dados são de domínio público, disponibilizadas pelo Instituto Brasileiro de Geografia e Estatística (IBGE).

A metodologia utilizada já foi previamente aplicada em estudos de mapeamento de pobreza e desigualdade ${ }^{19}$, desnutrição grave ${ }^{20}$ e $I A^{6,15}$. A metodologia determina a construção de um modelo de regressão logística em um inquérito nacional que, aplicado posteriormente ao censo demográfico do país, permite estimar um determinado desfecho em nível de desagregação menor.

Nesta pesquisa utilizou-se o Suplemento Segurança Alimentar da PNAD 2013, onde a IA foi aferida EBIA, composta de 14 perguntas, que avaliam a ocorrência de privação alimentar e fome no domicílio, nos últimos três meses. Cada resposta afirmativa recebe um ponto, e o score final classifica o domicílio em: 0 (segurança alimentar), 1-5 (insegurança leve), 6-9 (insegurança moderada) e 10-14 (insegurança grave) ${ }^{3}$. A IAG é caracterizada pela presença da fome nos domicílios, desfecho desta pesquisa ${ }^{5,6,15}$.

A amostra foi de 115.761 domicílios com EBIA completamente preenchida, excluindo-se os indígenas, domicílios coletivos e com pessoas de referência menores que 18 anos $^{15}$. Após a ava- 
liação da IA, os domicílios foram classificados dicotomicamente em: IAG (insegurança alimentar grave) e seguros (todos os demais). Iniciou-se então a fase de exploração das variáveis tradicionalmente associadas ao desfecho (IAG), mediante de regressão logística univariada, considerando-se a estrutura amostral complexa da PNAD $33,6,7,15,21,22$. Para escolha das variáveis, outro critério adotado foi a presença das mesmas no banco da amostra do Censo 2010. Essa etapa foi essencial para garantir aplicabilidade do modelo ao Censo. As variáveis com valor de $\mathrm{p}<0,05$ no teste de Wald foram selecionadas para serem testadas.

O modelo foi gerado utilizando regressão logística multivariada, sendo incluídas step-by-step às variáveis com maior associação com a IAG na análise univariada. As variáveis que permaneciam significativas ou que melhoravam poder de explicação do modelo eram mantidas. Foi verificado que não havia efeito de interação entre as UF e zonas urbano/rural na probabilidade de um domicílio ter IAG, sendo adotado modelo único ${ }^{6,15}$. Para avaliação da adequação do modelo foi utilizado Teste de Hosmer-Lemeshow, pseudo $\mathrm{R}^{2}$ e Curva ROC. O modelo final englobou as variáveis:

Ln $(\mathrm{p} \mathrm{IAG/1-p} \mathrm{IAG)}=-0,839-0,722(\mathrm{RO})+$ $0,640(\mathrm{AC})+0,672(\mathrm{AM})+0,384(\mathrm{RR})+0,594$ $(\mathrm{PA})+0,198(\mathrm{AP})-0,229(\mathrm{~T} 0)+0,514(\mathrm{MA})+$ 0,140 (PI) - 0,060 (CE) + 0,208 (RN) - 0,452 (PB) - 0,590 (PE) - 0,130 (AL) - 0,229 (SE) + 0,315 (BA) - 0,498 (MG) - 0,491 (ES) - 0,196 (RJ) 0,189 (SP) - 0,105 (PR) - 0,160 (RS) - 0,272 (MS) - 0,378 (MT) - 0,054 (GO) - 0,320 (DF) - 0,299 (Rural) + 0,452 (outra condição domicílio) + 0,299 (domicílio cedido) + 0,424 (domicilio alugado) - 0,860 (um banheiro) - 1,378 (dois banheiros) - 1,387 (três banheiros) - 2,132 (quatro banheiros) - 2,636 (cinco banheiros ou mais) + 0,217 (até 1/4 SMPC) - 0,470 (mais de $1 / 4$ até $1 / 2$ SMPC) - 1,091 (mais de $1 / 2$ até 1 SMPC) - 1,756 (mais de 1 até 2 SMPC) - 2,329 (mais de 2 até 3 SMPC) - 2,434 (mais de 3 até 5 SMPC) - 4,735 (mais de 5 SMPC) - 0,151 (chefe do domicílio idoso) $+0,332$ (chefe domicílio negro) - 0,571 (chefe domicilio com ensino fundamental completo e médio incompleto) - 0,967 (chefe do domicilio com ensino médio completo e superior incompleto) - 1,164 (chefe do domicílio com ensino superior completo) - 0,637 (vive em companhia de cônjuge ou companheiro) - 0,140 (não vive em companhia de cônjuge ou companheiro, mas já viveu) - 0,258 (trabalhou na semana de referência).

Após a determinação da equação preditiva, aplicou-se o modelo à base de dados da amos- tra do Censo 2010. Foi utilizada a amostra do Censo em virtude de maior disponibilidade de variáveis, comparada ao universo e consequente maior possibilidade de trabalhar-se com um modelo com melhor poder explicativo ${ }^{18}$. Cada variável do Censo 2010 foi codificada de forma exatamente igual à do modelo oriundo da PNAD 2013. O modelo aplicado gerou, então, a probabilidade de cada domicílio estar em situação de IAG. Depois desta etapa, utilizando a estrutura complexa da amostra do Censo, foi estimada a prevalência municipal de IAG para cada um dos 5.565 municípios brasileiros de 2010 .

Como a aplicação do modelo ocorreu nos dados do Censo de 2010, as estimativas geradas datam de 2010. Foi realizada a correção destas estimativas para o ano de 2013, utilizando um fator de correção municipal que consistia na razão das prevalências estaduais de IAG aferidas na PNAD 2013, e as estimadas para o ano de 2010. Isso permitiu que as estimativas municipais apresentadas sejam para o mesmo ano da última aplicação da EBIA em pesquisa nacional.

Os dados de prevalências municipais são apresentados em percentuais, amplitude e médias regionais e estaduais. Os municípios foram agrupados em categorias segundo quartis de prevalência de IAG: baixa (até 2,02\%), média (2,03 a $2,89 \%)$, alta (2,90 a $5,92 \%)$ e muito-alta (5,93 a 18,53\%). Essas mesmas categorias de prevalência de IAG foram utilizadas para a construção do mapa de distribuição espacial da IAG no Brasil, confeccionado utilizando-se o software QGIS Desktop 2.14.0.

Também são apresentados dados dos dez municípios com menores e maiores prevalências de IAG, assim como as maiores discrepâncias na prevalência municipal estimada em relação à média estadual aferida pela PNAD 2013. Discrepâncias positivas indicam quantos pontos percentuais o município ultrapassa a média estadual, e discrepâncias negativas indicam menores prevalências municipais em relação à média da UF.

As análises foram realizadas utilizando-se os softwares SPSS, versão 20.0 (SPSS Inc) e o software $\mathrm{R}$ (version 3.2.3). Em todas as fases da pesquisa foi considerada a estrutura complexa da amostra e utilizados os pesos amostrais recomendados.

\section{Resultados}

A adequação do modelo foi testada pelos testes de Hosmer-Lemeshow ( $\mathrm{p}=0,097)$, pseudo $\mathrm{R}^{2}(\mathrm{p}$ $=0,186)$ e curva ROC (área $=0,823)$. A prevalên- 
cia de IAG estimada pelo modelo, para o Brasil, foi de $3,21 \%$.

A Tabela 1 apresenta as prevalências de IAG segundo Região. Observa-se que a prevalência de IAG, calculada a partir das prevalências municipais, foi maior na Região Norte $(7,39 \%)$ e menor na Região Sudeste $(2,17 \%)$. Há grande heterogeneidade na distribuição das prevalências intra-regionais. A Região Nordeste foi a que apresentou maior discrepância nas prevalências de IAG em seus municípios, variando de 18,58\% a $0,83 \%$. Já a Região Sudeste foi a que apresentou maior uniformidade, com percentuais variando de $0,70 \%$ a $4,89 \%$ (Tabela 1 ).

A Figura 1 apresenta as médias e a variação estadual das prevalências de IAG nas UF brasi-

Tabela 1. Prevalência de insegurança alimentar grave em municípios brasileiros segundo região. Brasil, 2013.

\begin{tabular}{lcccc}
\hline Região & $\begin{array}{c}\text { Numero de } \\
\text { Municípios }\end{array}$ & $\begin{array}{c}\text { Média } \\
\text { IAG } \\
(\%)\end{array}$ & $\begin{array}{c}\text { Prevalência } \\
\text { Mínima de } \\
\text { IAG } \\
(\%)\end{array}$ & $\begin{array}{c}\text { Prevalência } \\
\text { Máxima } \\
\text { IAG } \\
(\%)\end{array}$ \\
\hline Norte & 449 & 7,39 & 1,27 & 17,81 \\
$\begin{array}{l}\text { Nordeste } \\
\text { Centro- }\end{array}$ & 1794 & 7,09 & 0,83 & 18,58 \\
Oeste & 1668 & 2,27 & 0,89 & 6,23 \\
Sudeste & 1188 & 2,17 & 0,70 & 4,89 \\
Sul & 466 & 2,86 & 1,33 & 7,58 \\
\hline
\end{tabular}

leiras. Observa-se que as maiores amplitudes de variação ocorreram nos Estados do Maranhão e Amapá. No Maranhão a prevalência municipal de IAG variou de $5,05 \%$ a $18,58 \%$ (dados não apresentados em tabelas). Já os Estados de Rio de Janeiro e Rondônia foram os que apresentaram menores variações nas prevalências municiais de IAG (Figura 1). Em Rondônia variaram de 1,28\% a 3,04\% (dados não apresentados em tabelas).

Com o intuito de melhor visualizar a distribuição espacial da IAG foi construído mapa (Figura 2) por quartis de prevalência de IAG. A cor mais clara evidencia baixas prevalências municipais (até 2,02\%). As cores vão se intensificando à medida que a prevalência de IAG aumenta, sendo os municípios com cor mais escura os com prevalência muito-alta de IAG (5,93\% a 17,81\%). Os municípios com prevalência muito-alta de IAG estão concentrados nas Regiões Norte e no semiárido do Nordeste brasileiro. As capitais da Região Norte, Manaus (AM, 5,05\%), Boa Vista (RR, 4,00\%), Macapá (AM, 3,37\%) e Belém (PA, $4,47 \%)$, tendem a apresentar menores prevalências (embora ainda altas) de IAG que seus municípios vizinhos. Observa-se que na Região Nordeste as prevalências de IAG nas zonas litorâneas é menor, exceto para municípios do Maranhão e Bahia. As prevalências de IA diminuem em direção ao Sul do país, com grande concentração de municípios com baixa prevalência de IA no sul de Minas Gerais, Região central do Mato Grosso,

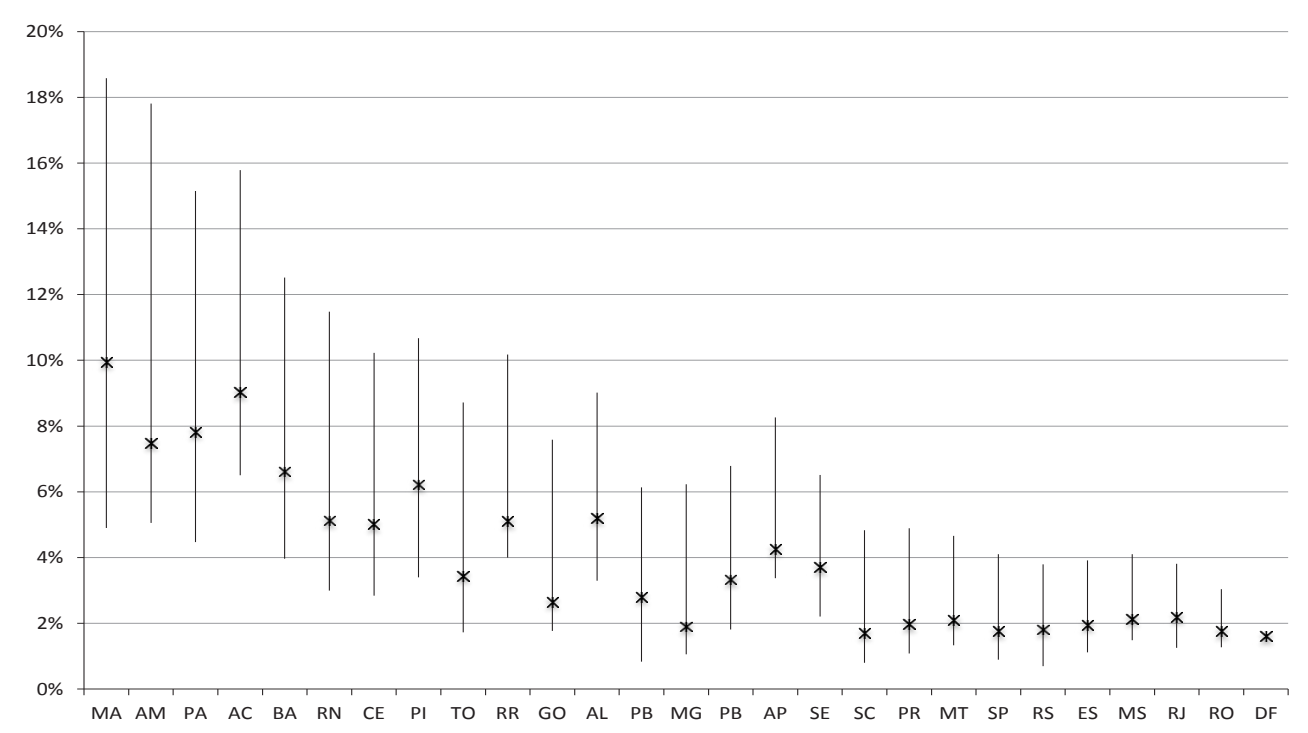

Figura 1. Variação estadual (em ordem decrescente) e média da prevalência de insegurança alimentar grave em municípios brasileiros. Brasil, 2013. 
$\operatorname{IAG}(\%)$

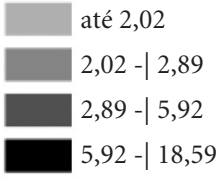

Figura 2. Distribuição espacial da Insegurança alimentar grave em municípios brasileiros, segundo quartil de prevalência. Brasil, 2013.

São Paulo, Santa Catarina e norte do Rio Grande do Sul (áreas mais claras do mapa).

A Tabela 2 apresenta a distribuição percentual dos municípios brasileiros, por Região e UF, segundo quartis de prevalência de IAG. Observa-se que apenas $0,3 \%$ dos municípios da Região Nordeste tem baixa prevalência de IAG, enquanto que na Região Sudeste este número é de $47,1 \%$. A Região Nordeste tem $62,7 \%$ dos seus municípios com prevalências muito-altas de IAG e a Região Sul não apresenta nenhum município nesta condição.

Essa mesma diferença na distribuição da IAG é observada entre UF brasileiras (Tabela 2). A presença de baixas e médias prevalências de IAG nas UFs da Região Norte e Nordeste brasileiras é nula ou mínima. Excetuando-se Rondônia, que apresenta prevalências de IA muito diferenciada dos seus pares das regiões Norte e Nordeste, o melhor desempenho é de Pernambuco, com
13,0\% dos seus municípios com prevalências baixas e médias. Acre, Amazonas, Pará, Maranhão e Bahia têm mais de $90 \%$ dos seus municípios com prevalências muito-altas de IAG. São Paulo, seguido de Santa Catarina e Rio Grande do Sul são os Estados com maior numero percentual de municípios com baixa prevalência de IAG.

A Tabela 3 apresenta os 10 municípios brasileiros com maiores e menores prevalências de IAG. O município com maior prevalência de IAG no Brasil é Serrano do Maranhão (MA), com $18,58 \%$ de IAG. Itamarati (AP) ocupa a segunda posição $(17,81 \%)$. Todos os outros oito municípios do ranking de maiores prevalências de IAG pertencem ao Estado do Maranhão. Dentre os dez municípios com menores prevalências de IAG no Brasil, sete estão localizados no Rio Grande do Sul, inclusive o município com menor prevalência de IAG: Nova Pádua, com $0,7 \%$ da população em IAG. Fazem parte desta lista, ain- 
Tabela 2. Categorias de prevalência de insegurança alimentar grave em municípios segundo Unidades da Federação. Brasil, 2013.

\begin{tabular}{|c|c|c|c|c|c|c|c|c|c|c|}
\hline \multirow[b]{2}{*}{ Região / UF } & \multicolumn{2}{|c|}{ Baixa $^{a}$} & \multicolumn{2}{|c|}{ Média $^{\mathrm{b}}$} & \multicolumn{2}{|c|}{ Alta $^{c}$} & \multicolumn{2}{|c|}{ Muito Alta ${ }^{\mathrm{d}}$} & \multicolumn{2}{|c|}{ Total } \\
\hline & $\mathbf{n}$ & $\%$ & $\mathbf{n}$ & $\%$ & $\mathbf{n}$ & $\%$ & $\mathbf{n}$ & $\%$ & $\mathbf{n}$ & $\%$ \\
\hline Norte & 23 & 5,1 & 36 & 8,0 & 132 & 29,4 & 258 & 57,5 & 449 & 100,0 \\
\hline Rondônia & 22 & 42,3 & 29 & 55,8 & 1 & 1,9 & 0 & * & 52 & 100,0 \\
\hline Acre & 0 & * & 0 & * & 0 & * & 22 & 100,0 & 22 & 100,0 \\
\hline Amazonas & 0 & * & 0 & * & 1 & 1,6 & 61 & 98,4 & 62 & 100,0 \\
\hline Roraima & 0 & * & 0 & * & 3 & 20,0 & 12 & 80,0 & 15 & 100,0 \\
\hline Pará & 0 & * & 0 & * & 6 & 4,2 & 137 & 95,8 & 143 & 100,0 \\
\hline Amapá & 0 & * & 0 & * & 8 & 50,0 & 8 & 50,0 & 16 & 100,0 \\
\hline Tocantins & 1 & 0,7 & 7 & 5,0 & 113 & 81,4 & 18 & 12,9 & 139 & 100,0 \\
\hline Nordeste & 5 & 0,3 & 33 & 1,8 & 631 & 35,2 & 1125 & 62,7 & 1794 & 100,0 \\
\hline Maranhão & 0 & * & 0 & * & 4 & 1,8 & 213 & 98,2 & 217 & 100,0 \\
\hline Piauí & 0 & * & 0 & * & 23 & 10,3 & 201 & 89,7 & 224 & 100,0 \\
\hline Ceara & 0 & * & 1 & 0,5 & 57 & 31,0 & 126 & 68,5 & 184 & 100,0 \\
\hline Rio Grande do Norte & 0 & * & 0 & * & 49 & 29,3 & 118 & 70,7 & 167 & 100,0 \\
\hline Paraíba & 1 & 0,4 & 11 & 4,9 & 207 & 92,9 & 4 & 1,8 & 223 & 100,0 \\
\hline Pernambuco & 4 & 2,2 & 20 & 10,8 & 160 & 86,5 & 1 & 0,5 & 185 & 100,0 \\
\hline alagoas & 0 & * & 0 & * & 28 & 27,5 & 74 & 72,5 & 102 & 100,0 \\
\hline Sergipe & 0 & * & 1 & 1,3 & 71 & 94,7 & 3 & 4,0 & 75 & 100,0 \\
\hline Bahia & 0 & * & 0 & * & 32 & 7,7 & 385 & 92,3 & 417 & 100,0 \\
\hline Sudeste & 785 & 47,1 & 613 & 36,7 & 269 & 16,1 & 1 & 0,1 & 1668 & 100,0 \\
\hline Minas Gerais & 368 & 43,1 & 268 & 31,5 & 216 & 25,3 & 1 & 0,1 & 853 & 100,0 \\
\hline Espírito Santo & 26 & 33,3 & 41 & 52,6 & 11 & 14,1 & 0 & * & 78 & 100,0 \\
\hline Rio de Janeiro & 10 & 10,9 & 62 & 67,4 & 20 & 21,7 & 0 & * & 92 & 100,0 \\
\hline São Paulo & 381 & 59,1 & 242 & 37,5 & 22 & 3,4 & 0 & * & 645 & 100,0 \\
\hline Sul & 543 & 45,7 & 464 & 39,1 & 181 & 15,2 & $\mathbf{0}$ & * & 1188 & 100,0 \\
\hline Paraná & 91 & 22,8 & 218 & 54,6 & 90 & 22,6 & 0 & * & 399 & 100,0 \\
\hline Santa Catarina & 171 & 58,4 & 81 & 27,6 & 41 & 14,0 & 0 & * & 293 & 100,0 \\
\hline Rio Grande do Sul & 281 & 56,7 & 165 & 33,2 & 50 & 10,1 & 0 & * & 496 & 100,0 \\
\hline Centro-Oeste & 50 & 10,7 & 232 & 49,8 & 182 & 39,1 & 2 & 0,4 & 466 & 100,0 \\
\hline Mato Grosso do Sul & 7 & 9,0 & 54 & 69,2 & 17 & 21,8 & 0 & * & 78 & 100,0 \\
\hline Mato Grosso & 36 & 25,5 & 77 & 54,6 & 28 & 19,9 & 0 & * & 141 & 100,0 \\
\hline Goiás & 6 & 2,4 & 101 & 41,1 & 137 & 55,7 & 2 & 0,8 & 246 & 100,0 \\
\hline Distrito Federal & 1 & 100,0 & 0 & * & 0 & * & 0 & * & 1 & 100,0 \\
\hline
\end{tabular}

da, os municípios de São Caetano do Sul (SP), Fernando de Noronha (PE) e Rio Fortuna em Santa Catarina.

As maiores discrepâncias percentuais positivas em relação à prevalência de IAG na UF (ou seja, o município apresenta IAG muito superior à da UF) ocorrem no Estado do Amazonas. O município com maior discrepância positiva é Itamarati (AM), que tem prevalência de IAG 10,32 pontos percentuais mais alta que a média do Estado. Já as menores discrepâncias negativas incluem quatro capitais (Salvador, Teresina, Belém e São Luís), demonstrando melhores condi- ções de IAG que os demais municípios nestas UF. A maior discrepância negativa foi observada em São Luís (MA), que teve prevalência 5,04 pontos percentuais mais baixa que a média do Estado (Tabela 3).

\section{Discussão}

O modelo criado para o ano de 2013 foi muito semelhante ao utilizado em 2004 para a predição de IAG(6). O modelo apresentou bom ajuste e adequada capacidade de predição, o que signi- 
Tabela 3. Municípios com maiores e menores prevalências de insegurança alimentar grave no Brasil e municípios com maiores discrepâncias percentuais de insegurança alimentar grave em relação à prevalência da Unidade da Federação. Brasil, 2103.

\begin{tabular}{|c|c|c|c|c|}
\hline Município & UF & $\begin{array}{c}\text { Prevalência } \\
\text { de IAG (\%) }\end{array}$ & $\begin{array}{l}\text { Prevalência Estadual } \\
\text { (PNAD 2013) (\%) }\end{array}$ & $\begin{array}{l}\text { Diferença }^{\star} \text { entre } \\
\text { prevalência } \\
\text { Municipal e da UF }\end{array}$ \\
\hline \multicolumn{5}{|l|}{ Maiores Prevalência de IAG } \\
\hline Serrano do Maranhão & MA & 18,58 & 9,94 & 8,64 \\
\hline Itamarati & $\mathrm{AM}$ & 17,81 & 7,48 & 10,32 \\
\hline Cachoeira Grande & MA & 17,09 & 9,94 & 7,15 \\
\hline Marajá do Sena & MA & 16,76 & 9,94 & 6,82 \\
\hline Presidente Juscelino & MA & 16,71 & 9,94 & 6,77 \\
\hline Afonso Cunha & MA & 16,48 & 9,94 & 6,53 \\
\hline Humberto de Campos & MA & 16,27 & 9,94 & 6,33 \\
\hline Belágua & MA & 16,10 & 9,94 & 6,16 \\
\hline Pedro do Rosário & MA & 16,02 & 9,94 & 6,07 \\
\hline Timbiras & MA & 16,01 & 9,94 & 6,06 \\
\hline \multicolumn{5}{|l|}{ Menores Prevalências de IAG } \\
\hline São José do Sul & RS & 0,90 & 1,80 & $-0,91$ \\
\hline São Caetano do Sul & SP & 0,89 & 1,76 & $-0,86$ \\
\hline Fernando de Noronha & $\mathrm{PE}$ & 0,83 & 2,81 & $-1,98$ \\
\hline Montauri & RS & 0,82 & 1,80 & $-0,98$ \\
\hline Carlos Barbosa & RS & 0,81 & 1,80 & $-0,99$ \\
\hline Rio Fortuna & SC & 0,80 & 1,70 & $-0,90$ \\
\hline União da Serra & RS & 0,80 & 1,80 & $-1,00$ \\
\hline Westfalia & RS & 0,78 & 1,80 & $-1,02$ \\
\hline Nova Candelária & RS & 0,74 & 1,80 & $-1,06$ \\
\hline Nova Pádua & RS & 0,70 & 1,80 & $-1,10$ \\
\hline \multicolumn{5}{|c|}{ Maiores Discrepâncias Positivas na Prevalência } \\
\hline Itamarati & $\mathrm{AM}$ & 17,81 & 7,48 & 10,32 \\
\hline Serrano do Maranhão & MA & 18,58 & 9,94 & 8,64 \\
\hline Santo Antonio do Içá & $\mathrm{AM}$ & 15,87 & 7,48 & 8,39 \\
\hline Jutaí & $\mathrm{AM}$ & 15,80 & 7,48 & 8,32 \\
\hline Guajará & $\mathrm{AM}$ & 15,45 & 7,48 & 7,97 \\
\hline Ipixuna & $\mathrm{AM}$ & 15,40 & 7,48 & 7,92 \\
\hline Beruri & $\mathrm{AM}$ & 15,19 & 7,48 & 7,71 \\
\hline Maraã & $\mathrm{AM}$ & 14,91 & 7,48 & 7,43 \\
\hline Envira & $\mathrm{AM}$ & 14,85 & 7,48 & 7,37 \\
\hline Cachoeira do Piriá & PA & 15,15 & 7,83 & 7,32 \\
\hline \multicolumn{5}{|c|}{$\begin{array}{l}\text { Maiores Discrepâncias Negativas na } \\
\text { Prevalência }\end{array}$} \\
\hline Salvador & BA & 3,96 & 6,62 & $-2,65$ \\
\hline Estreito & MA & 7,29 & 9,94 & $-2,65$ \\
\hline Açailândia & MA & 7,16 & 9,94 & $-2,78$ \\
\hline Teresina & PI & 3,40 & 6,22 & $-2,82$ \\
\hline Ananindeua & PA & 4,74 & 7,83 & $-3,09$ \\
\hline Novo Progresso & PA & 4,72 & 7,83 & $-3,11$ \\
\hline Belém & PA & 4,47 & 7,83 & $-3,36$ \\
\hline Paço do Lumiar & MA & 5,56 & 9,94 & $-4,38$ \\
\hline São José de Ribamar & MA & 5,36 & 9,94 & $-4,58$ \\
\hline Imperatriz & MA & 5,25 & 9,94 & $-4,70$ \\
\hline São Luís & MA & 4,90 & 9,94 & $-5,04$ \\
\hline
\end{tabular}


fica que os valores preditos não são diferentes dos verificados pela PNAD 2013. O valor predito pelo modelo para o Brasil foi de 3,21\%, mesmo valor aferido pela PNAD 2013 3 . As variáveis que permaneceram no modelo são tradicionalmente associadas à IAG e fome $\mathrm{e}^{6,7,10,11,21-23}$.

Os dados de prevalência municipal de IAG apresentados neste estudo fornecem informação importante para melhoria, fortalecimento e focalização dos programas sociais, politicas e ações de

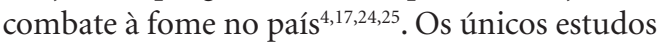
com representatividade municipal apontando prevalências municipais detalhadas IAG datam de 2003 e $2005^{7,10}$. Os outros estudos mais recentes apresentam dados de prevalências municipais agrupadas ou, ainda, agregam as categorias moderada e grave, não sendo possível a identificação exata da prevalência IAG em um município ${ }^{8,9}$. Outros, ainda, fazem recortes de populações específicas ${ }^{11,13,21,26}$. Os últimos dados de prevalências municipais para o Brasil (ainda que estimados por modelo preditivo) datam de $2004^{6}$.

Ainda em relação à fase de modelagem, é importante ressaltar o peso de cada um dos seus componentes na predição de IAG. Pode-se observar que o fator que mais fortemente influencia a probabilidade de ocorrência de IAG é renda, que apresenta os valores de mais elevados do modelo, mostrando uma associação forte entre renda e a ocorrência de IAG, o que já foi observado em estudos anteriores ${ }^{10,21-23}$. Segundo a PNAD 2013, famílias com renda entre $1 / 4$ a $1 / 2$ salários mínimos per capita (SMPC) apresentam prevalência de $28,6 \%$ de insegurança alimentar grave, enquanto famílias com renda superior a 2 SMPC apenas $2,2, \%{ }^{3}$. Fachini et al. ${ }^{23}$ verificaram que, na Região Nordeste, famílias com renda inferior a $R \$ 70,00$ tinham 8,38 vezes mais chances de sofrerem de IA moderada ou grave se comparadas àquelas com renda superior a $R \$ 300,00$, mesmo após controle de variáveis confundidoras.

A zona rural apresentou-se, na modelagem, como fator de proteção contra a IAG. Interessante ressaltar que normalmente a IAG é maior na zona rural se comparada à urbana ${ }^{3}$. Entretanto, no modelo , quando acrescidas as demais variáveis, morar na zona rural passa a ser fator de proteção, semelhante ao observado por Gubert et al. ${ }^{6}$.

A cor/raça do chefe do domicilio aparece ainda como importante componente da determinação da insegurança alimentar no Brasil. Estudos anteriores já haviam destacado a importância da questão étnico-racial na determinação da insegurança alimentar ${ }^{3,10}$, principalmente pelas maiores inequidades sociais observadas na população negra e parda no Brasil, quando comparada à população branca ${ }^{27}$. Negros e pardos representam mais de $50 \%$ da população brasileira ${ }^{27}$, entretanto são os com menor escolaridade e a minoria $(24,0 \%)$ no decil superior de renda no Brasil ${ }^{28}$.

A localização geográfica dos municípios também teve forte influencia na predição de IAG. A localização na Região Norte aumentava a chance de ocorrência de IAG (exceto para Tocantins). $\mathrm{Na}$ Região Nordeste a localização do município por vezes se caracterizava como fator de proteção (Ceará, Paraíba, Pernambuco, Alagoas e Sergipe) e outras como fator de risco para maior probabilidade de ocorrência de IAG (Maranhão, Piauí, Rio Grande do Norte e Bahia). Estar localizado nas Regiões Sul, Sudeste e Centro-Oeste sempre diminuiu a probabilidade de ocorrência de IAG.

Observa-se que as maiores prevalências de IAG municipais ocorreram na Região Norte, com muita heterogenia na sua distribuição, seguido pelo Nordeste. Em 2004 estas mesmas regiões apresentaram as maiores discrepâncias e, apesar de as prevalências terem diminuído, ainda são consideradas elevadas ${ }^{6}$. Fachinni et al. ${ }^{23}$ já haviam verificado diferenças regionais importantes na ocorrência de IA no Nordeste e Sul do Brasil. A ocorrência da IAG está intimamente ligada a determinantes econômicos e sociais. Enquanto a renda média habitual por pessoa ocupada na Região Norte era de R $\$ 1.540,00$, em 2015 , na Região Sul era de R\$2.183,00. Em 2015, a prevalência de pessoas sem instrução ou com menos de um ano de estudo na Região Nordeste era de 19,3\%, contra 8,4 na Região Sudeste ${ }^{29}$.

O Nordeste foi a Região com maiores discrepâncias na ocorrência de IA. Em 2004, essa discrepância foi de 29 pontos percentuais, enquanto que, em 2013, ela reduziu-se para 17,756. É importante considerar essas diferenças intra-regionais nas ações de combate à fome. Um município pertencer à Região Nordeste não o torna automaticamente prioridade nas ações de combate à fome, uma vez que nesta Região existem municípios com $0,85 \%$ de IAG e outros com prevalência de $18,58 \%$.

As Regiões Sul e Sudeste foram as que apresentaram menores prevalências de IAG e menores discrepâncias intra-regionais. Em termos de indicadores sociais, estas regiões são também as mais uniformes, justificando em parte estes resultados. O índice de Gini, por exemplo, na Região Sul é de 0,466, e 0,486, na Região Sudeste, valores abaixo da média Brasileira de 0,507, revelando menor desigualdade de renda ${ }^{30}$. 
Em relação à variação intra-estadual, a situação em 2013 foi em parte diferente da apresentada em 2004. Em 2004, a pior situação era apresentada por Maranhão e Rio Grande do Norte, que apresentaram as maiores amplitude na prevalência de IAG em seus municípios ${ }^{6}$. Em 2013, Maranhão seguiu encabeçando a lista, porém seguido de UFs da Região Norte (Amazonas, Amapá e Acre). Essa desigualdade observada na distribuição da IAG acompanha a desigualdade de geração de riqueza dos municípios da Região Norte. A participação acumulada dos 5 maiores municípios destes três estados para o Produto Interno Bruto estadual correspondeu a $87,5 \%$, $86,5 \%$ e $71,4 \%$, no ano de $2011^{31}$.

Avaliando a situação estadual, destaca-se o Estado de Roraima. Roraima apresenta prevalências menores de IAG que seus pares da Região Norte, bem como menor variação na ocorrência municipal. Roraima tem uma cobertura de 120,3 $\%$ da estimativa de famílias pobres no Estado inseridas no Programa Bolsa-Familia ${ }^{32}$. A UF também maior renda per capita no Estado ${ }^{29}$. Roraima ainda apresenta 100\% dos seus municípios com participação da Administração, saúde e educação públicas e seguridade social superior a $1 / 3$ do Produto Interno ${ }^{31}$. Tais fatores contribuem para a menor prevalência e variação de IAG observadas, uma vez que a IAG é intimamente associada a determinantes socioeconômicos ${ }^{3}$.

A análise espacial da distribuição espacial da IAG no Brasil mostra que, à medida que deslocase para o Sul, as prevalências de IAG se reduzem. De uma forma geral as prevalências de IAG são menores nas capitais das regiões Norte e Nordeste e, no Nordeste, os municípios da zona litorânea estão em melhor situação comparados aos do interior da Região. O interior na Região Nordeste é zona semiárida, com alta concentração de pobreza e escassez de recursos naturais, o que propicia ocorrência de IAG por fatores físicos, econômicos e sociais ${ }^{12,33}$. Ainda que estas regiões apresentem elevadas prevalências de IAG, houve uma redução expressiva nas prevalências entre 2004 e $2013^{6}$. Investimentos Federais no combate à pobreza extrema, como o programa Bolsa Família podem estar contribuindo para essa redução na IAG e maior acesso a alimentos ${ }^{34}$. Porém, independente destes esforços, é nítida a necessidade de homogeneizar essa ocorrência da IAG em percentuais mínimos, no país como um todo.

Entre as 10 maiores prevalências municipais, o Maranhão tem 9 de seus municípios. O Maranhão é também o Estado com maior numero de municípios com grande discrepância em relação à média municipal, sendo a UF com maior desigualdade na IAG no Brasil. Em 2004, o Maranhão também abrigava o município campeão em prevalência de IAG (Beláqua, na época com 31,1\% de IAG) ${ }^{6}$. Comparando-se a 2004, as prevalências diminuíram, entretanto, a UF permanece como a de pior situação entre todas as UFs brasileiras, em 2013. Reconhecidamente um Estado pobre, $\mathrm{O}$ Maranhão abriga 48,8\% dos seus munícipios entre os $10,0 \%$ mais pobres municípios brasileiros em relação ao PIB per capita ${ }^{31}$.

Já as menores prevalências de IAG municipais encontradas são predominantemente de municípios localizados no RS e SC. Chama a atenção Fernando de Noronha (PE), como um dos municípios com menor prevalência de IAG no Brasil. Entretanto trata-se de município com alto influxo monetário de recursos oriundos de atividades turísticas (serviços), assim como tem seu numero populacional controlado mediante rígidas regras de imigração, possuindo, em 2010, apenas 2630 moradores e Índice de Desenvolvimento Humano Municipal (IDHM) de 0,788, maior IDHM na UF ${ }^{18,35,36}$.

Esse estudo apresenta limitações metodológicas que devem ser consideradas. Apesar de o modelo construído apresentar bom poder de predição, tratam-se de dados estimados, podendo haver diferença em relação ao percentual real, caso a IAG fosse mensurada diretamente. Outra limitação é reduzido numero de variáveis disponíveis em ambos os bancos para a construção do modelo. Uma terceira limitação é a diferença temporal e periodicidade dos dados disponíveis, principalmente dados censitários (disponíveis a cada 10 anos), o que demandou correção das prevalências estimadas para o ano da pesquisa (2013).

\section{Conclusão}

Os municípios brasileiros apresentaram redução na IAG grave no período de 2004 a 2009, entretanto as prevalências de IAG permanecem elevadas. O país apresenta distribuição heterogênea da IAG no contexto intra-regional e intra-estadual, com grandes variações percentuais na prevalência de IAG dentro de um mesmo Estado ou Região. A IAG está mais concentrada em municípios das Regiões Norte e Nordeste, diminuindo à medida que se desloca para o Sul do país.

Os dados desta pesquisa podem auxiliar o processo de melhoria e focalização das políticas de combatem a fome no Brasil, uma vez que as 
prevalências estaduais (limite de desagregação disponível pela PNAD) muitas vezes ocultam situações municipais peculiares que merecem atenção nas ações públicas de promoção de segu- rança alimentar e nutricional. Estes achados ilustram o potencial valor da EBIA em ajudar na melhoria das ações e políticas públicas de combate à fome no Brasil, em nível nacional e municipal ${ }^{37}$.

\section{Colaboradores}

MB Gubert participou da concepção e planejamento, análise e interpretação dos dados, elaboração do rascunho, revisão crítica do conteúdo e participou da aprovação da versão final do manuscrito. R Perez-Escamilla participou da concepção e planejamento, interpretação dos dados, revisão crítica do conteúdo e participou da aprovação da versão final do manuscrito. 


\section{Referências}

1. Instituto de Pesquisa Econômica Aplicada (IPEA). Secretaria de Planejamento e Investimentos Estratégicos. Objetivos de Desenvolvimento do Milênio: Relatório $\mathrm{Na}$ cional de Acompanhamento. Brasília: Ipea; 2014.

2. Organização das Nações Unidas. Transformando nosso mundo: Agenda 2030 para o desenvolvimento Sustentável. [acessado 2016 Mar 29]. Disponível em: http:// www.pnud.org.br/Docs/Agenda2030completo_PtBR. pdf.

3. Instituto Brasileiro de Geografia e Estatística (IBGE). Pesquisa Nacional por Amostra de Domicílio: segurança alimentar 2013. Rio de Janeiro: IBGE; 2014.

4. Paes-Sousa R, Vaitsman J. The Zero Hunger and Brazil without Extreme Poverty programs: a step forward in Brazilian social protection policy. Cien Saude Colet 2014; 19(11):4351-4360.

5. Frongillo EA. Validation of measures of food insecurity and hunger. J Nutr 1999; 129(2S Supl.):506S-509S.

6. Gubert MB, Benicio MHD, Santos LMP. Estimates of severe food insecurity in Brazilian municipalities. Cad Saude Publica 2010; 26(8):1595-1605.

7. Vianna RPT, Segal-Correa AM. Insegurança alimentar das famílias residentes em municípios do interior do estado da Paraíba, Brasil. Rev Nutr 2008; 21(Supl.):111s122 s.

8. Cabral CS, Lopes AG, Lopes JM, Vianna RP. Food security, income, and the Bolsa Familia program: a cohort study of municipalities in Paraiba State, Brazil, 20052011. Cad Saude Publica 2014; 30(2):393-402.

9. Ferreira HS, Souza MEDCA, Moura FA, Horta BL. Prevalência e fatores associados à Insegurança Alimentar e Nutricional em famílias dos municípios do norte de Alagoas, Brasil, 2010. Cien Saude Colet 2014; 19(5):1533-1542.

10. Panigassi G, Segall-Corrêa AM, Marin-León L, Pérez-Escamilla R, Sampaio MFA, Maranha LK. Insegurança alimentar como indicador de iniqüidade: análise de inquérito populacional. Cad Saude Publica 2008; 24(10):2376-2384.

11. Souza NN, Dias MM, Sperandio N, Franceschini SCC, Priore SE. Perfil socioeconômico e insegurança alimentar e nutricional de famílias beneficiárias do Programa Bolsa Família no município de Viçosa, Estado de Minas Gerais, Brasil, em 2011: um estudo epidemiológico transversal. Epidemiol Serv Saúde 2012; 21(4):655-662.

12. Oliveira JS, Lira PIC, Maia SR, Sequeira LAS, Amorim RCA, Batista Filho M. Insegurança alimentar e estado nutricional de crianças de Gameleira, zona da mata do Nordeste brasileiro. Rev Bras Saúde Mater Infant 2010; 10(2):237-245.

13. Pedraza DF, Gama JS. Food and nutrition security in families with children under five years old in the city of Campina Grande, Paraiba. Rev Bras Epidemiol 2015; 18(4):906-917.

14. Peixoto MRG, Ramos K, Schingaglia RM, Braudes-Silva LA. Insegurança alimentar na área de abrangência do Núcleo de Apoio à Saúde da Família em Itumbiara, Goiás. Epidemiol. Serv. Saúde 2014; 23(2):327-336.

15. Gubert MB, Benicio MHD, Silva JP, Rosa TEC, Santos SM, Santos LMP. Use of a predictive model for food insecurity estimates in Brazil. Arch Latinoam Nutr 2010; 60(2):119-125.
16. Burlandy L. Construction of the food and nutrition security policy in Brazil: strategies and challenges in the promotion of intersectorality at the federal government level. Cien Saude Colet 2009; 14(3):851-860.

17. Custodio MB, Yuba TY, Cyrillo DC. Food and nutrition security policy in Brazil: an analysis of resource allocation. Rev Panam Salud Publica 2013; 33(2):144-150.

18. Instituto Brasileiro de Geografia e Estatística (IBGE). Censo Demográfico de 2010. Brasília: IBGE; 2011.

19. Elbers C, Lanjow JOE, Lanjouw P. Micro-level estimation of welfare. Policy Research Working Paper, n. 2911. Washington: World Bank; 2002.

20. Benicio MHDA, Venancio SI, Konno SC, Monteiro CA. Novas estimativas para a prevalência de desnutrição na infância nos 5.507 municípios brasileiros a partir de modelos logísticos multinivel aplicados à amostra de crianças do Censo 2000. São Paulo: Universidade de São Paulo; 2005. (Série Pesquisas em Epidemiologia Nutricional, 1/05).

21. Poblacion AP, Marin-Leon L, Segall-Correa AM, Silveira JA, Taddei JA. Food insecurity in Brazilian families with children under five years of age. Cad Saude Publica 2014; 30(5):1067-1078.

22. Salles-Costa R, Pereira RA, Vasconcellos MTLD, Veiga GVD, Marins VMRD, Jardim BC, Gomes FS, Sichieri R. Associação entre fatores socioeconômicos e insegurança alimentar: estudo de base populacional na Região Metropolitana do Rio de Janeiro, Brasil. Rev Nutr 2008; 21(Supl.):99s-109s.

23. Facchini LA, Nunes BP, Motta JV, Tomasi E, Silva SM, Thume E, Silveira DS, Siqueira FV, Dilélio AS, Saes Mde O, Miranda VI, Volz PM, Osório A, Fassa AG. Food insecurity in the Northeast and South of Brazil: magnitude, associated factors, and per capita income patterns for reducing inequities. Cad Saude Publica 2014; 30(1):161-174.

24. Alves KP, Jaime PC. The national food and nutrition policy and its dialogue with the national food and nutrition security policy. Cien Saude Colet 2014; 19(11):4331-4340.

25. Schmitz BA, Moreira EA, Freitas MB, Fiates GM, Gabriel CG, Fagundes RL. Public intervention in food and nutrition in Brazil. Arch Latinoam Nutr 2011; 61(4):361-366.

26. Oliveira JS, Lira PIC, Andrade SLLS, Sales AC, Maia SR, Batista Filho M. Insegurança Alimentar e estado nutricional de crianças de São João do Tigre, no semi-árido do Nordeste. Rev Bras Epidemio 2009; 12(3):413-423.

27. Instituto Brasileiro de Geografia e Estatística (IBGE). Atlas do Censo Demofrafico 2010. Rio de Janeiro: IBGE; 2013.

28. Instituto de Pesquisa Econômica Aplicada (Ipea). Retrato das desigualdades de gênero e raça. $4^{\mathrm{a}}$ ed. Brasília: Ipea; 2011.

29. Instituto Brasileiro de Geografia e Estatística (IBGE). Pesquisa Nacional por Amostras de Domicílios Contínua - PNAD Contínua. 2015. [acessado 2016 Abr 30]. Disponivel em: http://www.ibge.gov.br/home/estatistica/ pesquisas/ pesquisa_resultados.php?id_pesquisa $=149$

30. Instituto Brasileiro de Geografia e Estatística (IBGE). Sintese de Indicadores Sociais. Uma análise da população Brasileira - 2013. Rio de Janeiro: IBGE; 2013. 
31. Instituto Brasileiro de Geografia e Estatística (IBGE). Produto Interno Bruto dos Municípios 2011. Contas Nacionais número 41. Rio de Janeiro: IBGE; 2013.

32. Brasil. Ministério do Desenvolvimento Social e Combate à Fome. Relatórios de Informações Sociais. [acessado 2016 Abr 11]. Disponível em: http://aplicacoes.mds. gov.br/sagi/RIv3/geral/relatorio.php\#Estimativas.

33. Rocha EMB, Lima RT, Almeida PC. Insegurança alimentar relacionada à área de residência em município do Semiárido brasileiro. Cad Saúde Colet 2014; 22(2):205-211.

34. Martins APB, Canella DS, Baraldi LG, Monteiro CA. Transferencia de renda no Brasil e desfechos nutricionais: revisao sistematica. Rev Saude Publica 2013; 47(6):1159-1171.

35. Pernambuco. Governo do Estado. Lei No 11.304, de 28 de dezembro de 1995. Institui o Distrito Estadual de Fernando de Noronha, aprova a sua Lei Orgânica, dispõe sobre medidas de natureza administrativa e dá outras providências. Diário Oficial do Estado de Pernambuco 1995; 29 dez.

36. Programa das Nações Unidas para o Desenvolvimento (PNUD). Atlas Brasil 2013. [acessado 2016 Abr 1]. Disponível em: http://www.atlasbrasil.org.br/2013/.

37. Perez-Escamilla R. Can experience-based household food security scales help improve food security governance? Glob Food Sec 2012; 1(2):120-125.

Artigo apresentado em 14/06/2016

Aprovado em 05/10/2016

Versão final apresentada em 07/10/2016 